\title{
An approach to comparing different ontologies in the context of hydrographical information
}

\author{
L. M. Vilches-Blázquez ${ }^{1}$, J. A. Ramos², F. J. López-Pellicer³ \\ O. $\mathrm{Corcho}^{4}$, J. Nogueras-Iso ${ }^{5}$ \\ 1, 2, 4 Ontology Engineering Group - Universidad Politécnica de Madrid. Spain \\ ${ }^{3,5}$ Computer Science and Systems Engineering Department, University of Zaragoza. Spain \\ $\left\{{ }^{1}\right.$ lvilches, ${ }^{2}$ jarg, ${ }^{4}$ ocorcho\}@fi.upm.es \\ $\left\{{ }^{3}\right.$ fjlopez, ${ }^{5}$ jnog $\} @$ unizar.es
}

\begin{abstract}
Geographical Information is increasingly captured, managed and updated by different cartographic agencies. This information presents different structures and variable levels of granularity and quality. In practice, such heterogeneity causes the building up of multiple sets of geodata with different underlying models and schemas that have different structure and semantics. Ontologies are a proposal widely used for solving heterogeneity and a way of achieving the data harmonization and integration that GIS and SDI need.

This paper presents three hydrographical ontologies (which are built using topdown and bottom-up approaches) and an approach to comparing them; the goal of this approach is to prove which ontologies have a better coverage of the domain. In order to compare the resultant ontologies, six qualitative facets have been studied: sources used (amount, richness and consensus), reliability of building approaches (community extending use, recommendations), ontology richness (number and types of components), formalization (language), granularity (scale factor) and the design criteria followed.
\end{abstract}

Keywords: Geographical Information, heterogeneity, data harmonization and integration, top-down and bottom-up approach, hydrographical ontologies, comparison.

\section{Introduction}

Nowadays, Geographical Information (GI) is increasingly captured, managed and updated by different cartographic agencies. Such information presents different structures and variable levels of granularity and quality. In practice, this diversity causes the building up of multiple sets of spatial data and models with different structure and underlying semantics.

Ontologies are frequently used to describe explicitly the characteristics of these sets and models in a formal manner. They are a widespread proposal for solving heterogeneity and a way of achieving the data harmonization and integration that Geographical Information Systems (GIS) and Spatial Data Infrastructures (SDI) need to access, exchange and query processes. Domain ontologies $[22,28]$ are reusable in a 
specific domain. They provide vocabularies of concepts within a domain and their relationships, of the activities taking place in that domain, and of the theories and elementary principles governing that domain.

However, not a single ontology is devoted to the hydrographical domain, but many. Hydrography focuses on the measurement and description of the characteristics of any type of water bodies. Hydrography is composed of subsets, which are called subdomains, and these can be as different as oceanography, bathymetry, limnology, or hydrographical survey. This variety of subdomains justifies the need to specify some criteria for ontology comparison. This comparison, on the other hand, will allow other ontology or application developers to determine which ontology has a better conceptualization of the domain, though its selection process depend on usability criteria, language restrictions and others. Therefore, in this paper we aim to set an approach to comparing and evaluating the coverage of different domain ontologies.Nevertheless, we must make clear that this paper does not deal with processes that measure the similarity between concepts of different ontologies or that achieve an evaluation process of internal ontology characteristics (taxonomic correctly, consistency, cycle existence, etc.).

This paper is organized as follows. Section 2 describes the ontology development process. Section 3 presents two bottom-up approaches (Hydro and PhenomenOntology) and a top-down approach (hydrOntology) for ontology development. Section 4 describes some criteria for comparing and a comparison process between different ontologies. Finally, Section 5 draws some brief conclusions.

\section{Ontology development process}

As described in [27] there are two main different strategies for ontology development depending on the approach followed to obtain the ontology components and to represent them in the ontology. These are the bottom up and the top-down strategies and both have advantages and disadvantages.

The bottom-up strategy proposes identifying first the most specific concepts and generalizing them into more abstract concepts. The authors affirm that a bottom-up approach provides a very high level of detail. This approach (1) increases the overall effort (sustained by people and machines), (2) makes it difficult to spot commonality between related concepts, and (3) increases the risk of inconsistencies, which can lead to (4) remaking the strategy and even to more effort.

On the other hand, when the top-down strategy is used, the most abstract concepts are first identified, and then specialized into more specific concepts. The main result of using this approach is a better control of the level of detail; however, starting at the top may suppose having to choose and later impose arbitrary and possibly unnecessary high level categories. Because these categories do not arise naturally, we risk having less stability in the model, which may imply having to remake the model and also greater effort. The emphasis on dividing up rather than on putting together 
the categories implies, for a different reason, missing the commonality inherent in the complex web of interconnected concepts.

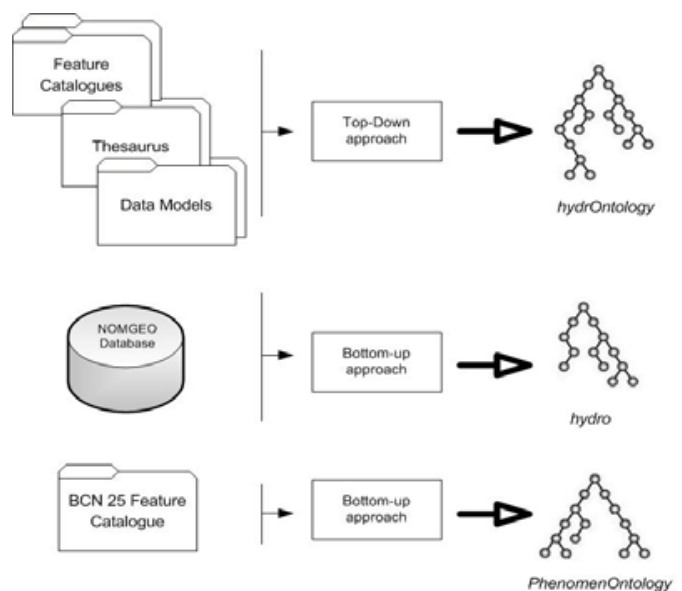

Figure 1. An overview of the three ways of developing hydrographical ontologies

Figure 1 shows an overview of the different manners of developing hydrographical ontologies following these approaches. The ontologies, which are described in detail in the next section, are built following different methods and methodologies.

\section{Ontologies for the hydrographical domain}

In this section we briefly describe some technical and methodological characteristics (bottom-up, top-down, etc.) of three ontologies belonging to the hydrographical domain.

\section{1 hydrOntology}

hydrOntology is an ontology that follows a top-down development approach. Its main goal is to harmonize heterogeneous information sources coming from diverse cartographic agencies and other international resources.

Initially, this ontology was created as a local ontology that established mappings between different Spanish National Geographic Institute (IGN-E) data sources (feature catalogues, gazetteers, etc.). Its purpose was to serve as a harmonization framework among Spanish cartographic producers. Later, the ontology has evolved into a global domain ontology and it attempts to cover most of the hydrographical domain. The final version of this ontology was finished in the mid-2008.

An overview of the hydrOntology characteristics with the statistical data (metrics) and its different taxonomic relations is provided below.

hydrOntology has 150 classes, 34 object properties, 66 data properties and 256 axioms. Some examples of the four taxonomic relations defined in the Frame 
Ontology [6] and the OKBC Ontology [3], namely, Subclasses, DisjointDecomposition, Exhaustive-Decomposition and Partitions have been implemented. Further details are shown in [29]. The ontology documentation is exhaustive and, in this sense, definitions and their sources can be found in each concept (class). The ontology has an important amount of labels with alternative names (synonyms) as well as concept and synonyms provenances.

In order to develop this ontology following a top-down approach, different knowledge models (feature catalogues of the IGN-E, the Water Framework European Directive, the Alexandria Digital Library, the UNESCO Thesaurus, Getty Thesaurus, GeoNames, FACC codes, EuroGlobalMap, EuroRegionalMap, EuroGeonames, different Spanish Gazetteers and many others) have been consulted; additionally, some integration problems of geographic information and several structuring criteria [29] have been considered. The aim was to cover most of the existing GI sources and build an exhaustive global domain ontology. For this reason, the ontology contains one hundred and fifty (150) relevant concepts related to hydrography (e.g. river, reservoir, lake, channel, and others).

Regarding methodological issues, the approach adopted is METHONTOLOGY, a widely-used ontology building methodology. This methodology emphasises the reuse of existing domain and upper-level ontologies and proposes using, for formalisation purposes, a set of intermediate representations that can be later transformed automatically into different formal languages.

hydrOntology has been developed according to the ontology design principles proposed by [14] and [2]. Some of its most important characteristics are that the concept names (classes) are sufficiently explanatory and are rightly written. Thus each class tries to group only one concept and, therefore, classes in brackets and/or with links ("and", "or") are avoided. According to some naming conventions, each class is written with a capital letter at the beginning of each word, while object and data properties are written with lower case letters.

\subsection{Hydro}

This bottom-up hydrographical ontology, developed at the University of Zaragoza (its final version appeared in 2007), is the result of applying the process described in [20] to the hydrographical features of the National Geographic Gazetteer, also called Georeferenced Database or NOMGEO. Such a process consists in applying to the toponymic database the following tasks: 1) the thematic analysis task, which determines the feature type and the generic name of each feature in the database; 2) the signature filter task, which selects the distinct signatures, i.e., pairs made of a feature type and a generic name that represents a significant number of features; 3 ) the formal context builder task, which creates an incidence matrix whose rows are the aforesaid signatures. Once the incidence matrix is built, the lattice builder task applies Formal Concept Analysis (FCA) techniques to produce a concept lattice; and 4) the ontology generator task, which transforms that lattice into OWL (Web Ontology Language).

The National Geographic Gazetteer is the source used for building Hydro. The gazetteer compiles more than 495,000 toponyms classified in 82 feature types. These 
toponyms have been drawn from several national and provincial atlas and gazetteers, cartographical maps and specialized toponymic databases. To build Hydro, six feature types with 110,000 toponyms were selected. It should be explained that this gazetteer has underlying a geographical database, not a lexical one. It contains a huge collection of proper names used to identify features in the real world and the type of feature and also some hint of location in the form of a pair of coordinates.

With regard to richness, this ontology contains 51 named classes, which can be classified from their source as follows: the original IGN-E feature types and the types extracted from the name of the features, i.e. generic names, and also the concepts derived from the combination (map) of concepts in the previous groups. It must be added that this ontology is more comprehensive than the six original hydrographical feature types. However, it is also more difficult to interpret because its correspondence in terms of the real world depends on the interpretation of terms used in the database - mostly generic names - and on the semantics of the concepts generated by FCA. Sometimes FCA generates concepts that exist but that cannot be immediately interpreted by the expert.

With respect to the methodology, this approach is based on the FCA [7]. Further details of this methodology and of its different steps of building process are shown in [20]. The ontology follows some design principles regarding the names of the classes, and these principles are the names drawn from the original feature type names, or from the generic names, or from a composite name made by joining both a feature type and a generic name.

\subsection{PhenomenOntology}

During 2007, the Ontology Engineering Group (at the Universidad Politécnica de Madrid) and IGN-E collaborated in building automatically an ontology of geographic features; their aim was to integrate the knowledge bases of this geographic agency. Both partners decided to use BCN25 (Numerical Cartographic Database scale $1: 25,000)$ to create the ontology automatically. The current version of PhenomenOntology is 2.0 and dates from September 2008.

The Numerical Cartographic Database (BCN25) was built to obtain the 1:25,000 cartographic information that complies with the required data specifications exploited inside Geographic Information Systems (GIS) environments. Therefore, BCN25 contains geometric and topological properties and follows a specific database oriented model and feature catalogue [25]. The information contained in BCN25 is structured in eight different topics (Administrative boundaries, Relief, Hydrography, Vegetation, Buildings, Communications, Piping lines and Toponymy). Each topic is coded with three pairs of digits: two digits for its topic, two digits for its group (part of homogeneous information structured in topics) and two digits for its subgroup (a stretch of geographic feature belongs to a group). These numbers describe and classify different features regardless of its location and spatial dimension. The following text box shows an example:

Topic 03: Hydrography

Group: 01 constant watercourse

Subgroup: 01 Watercourse symbolized with one line 
The authors have used a 1:25,000 scale catalogue to generate automatically an ontology using an ad hoc application. Such an application extracts the concepts of PhenomenOntology from the feature type attributes found in the instances of a feature catalogue. The application is a wrapper that upgrades the information presented as a collection of rows to a taxonomy of concepts order.

The software developed for the automatic creation of this ontology permits selecting the criteria for taxonomy creation and its order. These criteria are based on the information contained in each row of the BCN25 feature catalogue table. The code ("código") column stores encoded information about a three-level taxonomy. The application permits extracting a super class for each different value of the pair of digits selected.

IGN-E used the application developed for generating criteria combination tests and chose an automatic generated ontology with three levels (two criteria: first pair of code and second pair of code), 686 concepts and 3,846 attributes. In this paper, we focus only on the hydrographical subtree, which contains 87 concepts and 468 attributes (416 attributes corresponded directly to table columns, 35 were automatically generated in superclasses with criteria information and 17 were discovered in name analysis). The ontology is stored in the WebODE platform [1]. This first version of PhenomenOntology is being refined by IGN-E experts with the WebODE Editor. The subtree includes 52 concept names taken from BCN25 names (with its naming convention), 34 concept names, which are composed of a second pair of code, and one concept name composed of a first pair of code (03).

\section{Comparison}

By providing a shared conceptualization of a domain of interest, ontologies have become an important means for knowledge interchange and integration. However, up to date, there are not many works whose aim is to compare ontologies in the same domain. Different approaches make this comparison, which is based on matching techniques [23, 5], comparisons to conceptual level [33], similarity measurements [32, 21 and 9] and senses refinement algorithms [31, 30].

As we mentioned in the introduction, the goal of this paper is not to measure the similarity of ontologies or of ontology parts, neither is to set alignments with different matching tools (in fact, matching techniques do not achieve good results); in fact, our work is focused on the facets cited.

On the other hand, no criteria exist that evaluate domain coverage. There are only evaluation criteria, such as $[11,12,16]$, which focus on ontology verification, ontology validation, ontology assessment, consistency and philosophical notions of properties and concepts. Hence, we have to compare different ontologies on the same knowledge domain and then propose some evaluation criteria to point out which ontology better covers the domain, which, in this case, is the hydrographical domain.

The selected criteria for making comparisons between different ontologies follow some of the general ontology design criteria published in the literature; these criteria also focus on the different aspects covered by the most widely used ontology definition, i.e., an ontology is a formal, explicit specification of a shared 
conceptualisation [15]. Finally, these criteria take into account some aspects of the methodological process for building the ontologies. Therefore, in this paper we group different selected criteria and emphasize some concepts of the commented ontology definition. Thus, the significance of this definition is used as a basis for our proposal of comparison features. According to [26], a "conceptualisation" refers to an abstract model of some phenomenon in the world by having identified the relevant concepts of that phenomenon. "Explicit" means that the type of concepts used, and the constraints on their use are explicitly defined. "Formal" refers to the fact that the ontology should be machine readable, which excludes natural language. "Shared" reflects the notion that an ontology captures consensual knowledge, that is, knowledge that is not exclusive to some individuals, but accepted by a group. The comparison criteria followed, which are detailed in the next section, are the following: ontological richness and reliability of methodologies as regards conceptualization; sources and granularity with respect to shareability; implementation tools and formalization language with respect to explicitness and formality; and design criteria.

\subsection{Conceptualization quality}

Ontological richness. These ontologies contain information sources with different quality and granularity characteristics, and this divergence creates their different ontological richness, i.e., different components, which can change both in number and type. PhenomenOntology has 87 different classes and only 17 data properties because various identifiers (IDs) are not considered as real data properties in this comparison process since such identifiers were created during the automatic development of the ontology. Hydro has only classes (51 different classes) because of the method used to develop this ontology, whereas hydrOntology has 150 classes, 34 object properties, 66 data properties and 256 axioms; this difference is in part due to the amount and granularity of the sources selected for its development and to the information processing and modelling steps followed by the domain experts.

Reliability of methodologies. hydrOntology was developed with the help of METHONTOLOGY, a methodology that has been applied by different groups to build ontologies in different knowledge domains, such as Chemistry, Science, Knowledge Management, e-Commerce, etc. A detailed description of this methodology can be found in [10]. METHONTOLOGY takes into account the main activities identified by the IEEE software development process [18] and other knowledge engineering methodologies. METHONTOLOGY has been proposed as the methodology for ontology construction by the Foundation for Intelligent Physical Agents (FIPA), which promotes inter-operability across agent-based applications. On the other hand, Hydro is based on FCA [7] and follows a bottom-up approach. This ontology has proven to be very useful in different steps of the ontology building process. It is used during the ontology extraction step and in fields as different as medicine [19] and software development [17]. METHONTOLOGY can be found in data-driven ontology building scenarios, such as [8]. Finally, PhenomenOntology is developed following an ad hoc methodology. Obviously, the reliability of the methodologies is much higher than the reliability of the ad hoc wrapper. 


\subsection{Shareability}

Sources. Both bottom-up approaches are based on one available information source, respectively. Thus, PhenomenOntology is based on the BCN25 feature catalogue, whereas Hydro is based on the National Geographic Gazetteer. On the other hand, the top-down approach (hydrOntology) is based on more than 20 different information sources, as commented above. This fact implies that the building of hydrOntology uses a great amount of shared concepts. These ontologies have many differences between them with respect to the level of detail, as shown in Table 1 .

Table 1. Criteria for comparing ontologies

\begin{tabular}{|l|c|c|c|c|c|}
\cline { 2 - 6 } \multicolumn{1}{c|}{ hichness } & Sources & Methodology & Granularity & Formalization \\
\hline Hydro & $\begin{array}{c}150 \text { classes } \\
34 \text { object } \\
\text { properties } \\
66 \text { data } \\
\text { properties } \\
256 \text { axioms }\end{array}$ & $\begin{array}{c}\text { different } \\
\text { ones }\end{array}$ & $\begin{array}{c}\text { METHON- } \\
\text { TOLOGY }\end{array}$ & $\begin{array}{c}\text { From Global- } \\
\text { Continental to } \\
\text { Regional- } \\
\text { Local }\end{array}$ & OWL Full \\
\hline $\begin{array}{l}\text { Phenomen } \\
\text { Ontology 2.0 }\end{array}$ & $\begin{array}{c}\text { 87 classes } \\
\text { 17 data } \\
\text { properties }\end{array}$ & $\begin{array}{c}\text { National } \\
\text { Geographic } \\
\text { Gazetteer }\end{array}$ & FCN25 & $\begin{array}{c}\text { Ad hoc } \\
\text { wrapper }\end{array}$ & $1: 25,000$ to \\
$1: 5,000$ & OWL Full \\
\hline
\end{tabular}

Granularity. According to [36], the granularity concept is shared by two different viewpoints. In the first one, semantic granularity addresses the different levels of specification of an entity in the real world. In the second viewpoint, spatial granularity addresses the different levels of spatial resolution or representation at different scales. In this paper we deal with a merged vision of both viewpoints; therefore, the notion of granularity applied to GIS should take into account some cognitive aspects, the amount of detail involved in the presence-absence, and a representation of different geographical features across a wide range of scales.

With regard to the three ontologies that are the target of this comparing process, it should be said that they have different levels of granularity; therefore, hydrOntology, Hydro and PhenomenOntology are fed on different information sources, whereas certain phenomena are scale-dependent. Thus, PhenomenOntology is based on one source with geographical features, whose detail level corresponds to a 1:25,000 scale. Hydro is also fed on one source that has information of instances from a 1:25,000 to a 1:5,000 scale. hydrOntology, which is based on more than twenty different sources, has geographical features that range from a global-continental scale to a regional-local scale. Accordingly, these variations at the level of information detail serve to classify ontologies into two levels: Low-level ontologies, which correspond to very detailed information (hydrOntology), and high-level ontologies, which correspond to more general information [36] (Hydro and PhenomenOntology). 


\subsection{Explicity and Formality}

Implementation tools. The tools employed for developing these ontologies guarantee that all their components are explicit and formalized. hydrOntology and Hydro employ Protégé [24], while PhenomenOntology employ WebODE [1].

Formalization language. The three different ontologies are formalized according to Web Ontology Language (OWL) [34]. This language is a technical recommendation of the W3C Consortium ${ }^{1}$, and from such a language three increasingly expressive sublanguages (OWL Lite, OWL DL, and OWL Full) [35] have derived. Next, we show briefly some characteristics of these sublanguages. Further details can be found on the W3C website $^{2}$.

- OWL Lite gives support primarily to those users that require a classification hierarchy and simple constraints. OWL Lite has a less formal complexity than OWL DL.

- OWL DL helps those users who want maximum expressiveness while retaining computational completeness (all conclusions are guaranteed to be computable) and decidability (all computations will finish in finite time). This sublanguage includes all OWL language constructs, though these can be used only under certain restrictions. PhenomenOntology is formalized according to the expressiveness of this language.

- OWL Full is meant for users who need maximum expressiveness of and syntactic freedom from the Resource Description Framework ${ }^{3}$ (RDF) with no computational guarantees. OWL Full allows an ontology to augment the meaning of the predefined (RDF or OWL) vocabulary. It is very unlikely that any reasoning software can support complete reasoning for every feature of OWL Full. Hydro and hydrOntology are formalized according to the characteristics of this sublanguage.

\subsection{Design criteria}

The following criteria have been taken into account in our comparison process:

1. Clarity criterion [13]: An ontology should communicate effectively the intended meaning of the defined terms. These definitions should be objective and documented with natural language.

1Ext. Extended criterion [4]: Term names should be sufficiently descriptive and be used and written correctly, even if there are not definitions

2. Extendibility criterion [13]: One should be able to define new terms for special uses based on the existing vocabulary in a way that it does not require the revision of the existing definitions.

3. Minimizing the syntactic distance between sibling concepts criterion [2]: sibling concepts should be represented using the same primitives.

4. Standardization of names criterion [2]: The same naming conventions should be used to name related terms to ease the understanding.

\footnotetext{
${ }^{1}$ http://www.w3.org/

${ }^{2}$ http http://www.w3.org/TR/owl-features/

${ }^{3}$ http://www.w3.org/RDF/
} 
5. Minimal encoding bias criterion [14]: Design decisions should be independent of the implementation features.

6. Minimal presentation bias criterion [4]: Design decisions should be independent of the presentation features.

7. Maximizing the useful information quantity criterion [4]: The information of an ontology should be complete and should not have redundancies for being useful.

Table 2. Design criteria for comparing ontologies

\begin{tabular}{|l|c|c|c|c|c|}
\cline { 2 - 6 } \multicolumn{1}{c|}{} & $\mathbf{1}$ & $\mathbf{1}$ Ext. & $\mathbf{3}$ & $\mathbf{4}$ & $\mathbf{6}$ \\
\hline hydrOntology & $\checkmark$ & $\checkmark$ & $\checkmark$ & $\checkmark$ & $\checkmark$ \\
\hline Hydro & $\checkmark$ & X & $\sim$ & $\checkmark$ & $\checkmark$ \\
\hline PhenomenOntology & BCN25 & X & $\sim$ & $\checkmark$ & $\checkmark$ \\
\hline
\end{tabular}

As can be seen in Table 2, criteria 2 and 5 are not applicable, whereas criterion 7 is evaluated as ontology richness (see section 4.1). Moreover, the naming convention of PhenomenOntology is the same as that of its source (BCN25) because the wrapper does not implement any name transformation. As for the extended design criterion 1 , we must add that only hydrOntology observes it. With regard to criterion 3, it can be said that Hydro and PhenomenOntology present some sets of siblings.

\section{Conclusions and future work}

We have presented some criteria for ontology comparison that will permit ontology or application developers to investigate which ontology has a better domain conceptualization. The ontology comparison task using the above criteria is a complex one. As it can be observed, the ontological richness, the sources used, the methodological approach and the design criteria of ontologies of the same domain are very different. This reflects the fact that ontology development in a domain is strongly biased by the point of view of the experts involved, hence the need to establish some ontology comparison criteria previous to reusing an existing ontology.

With regard to future works, we are attempting to collect some hydrographical ontologies in order to carry out an exhaustive comparison and then to choose the ontology that covers best this knowledge domain. Additionally, we are trying to test our approach by means of comparing other ontologies of different geospatial topics among themselves and with other domain ontologies out of the geographical domain. Moreover, we will analyze other criteria for comparison process between ontologies. The goal of our approach is to set such an approach as an international comparison method of domain coverage.

\section{Acknowledgments}

This work has been partially supported by the National Projects "GeoBuddies: Anotación semántica colaborativa con dispositivos móviles en el Camino de Santiago" (TSI2007-65677C02) and "Avances en la definición y tratamiento de 
metainformación para la gestión de información y servicios en infraestructuras de datos geoespaciales" (TIN2007-65341), and the bilateral collaboration UPM - IGN-E 2007-2008. We are also grateful to Rosario Plaza for her help in checking and reviewing the grammar and spelling of the paper and improving clarity and style.

\section{References}

[1] Arpírez JC, Corcho O, Fernández-López M, Gómez-Pérez A (2003) "WebODE in a nutshell”. AI Magazine, 2003.

[2] Arpírez JC, Gómez-Pérez A, Lozano A, Pinto HS (1998) (ONTO)2Agent: An ontologybased WWW broker to select ontologies. In: Gómez-Pérez A, Benjamins RV (eds) ECAI’98 Workshop on Applications of Ontologies and Problem-Solving Methods. Brighton, United Kingdom, pp 16-24

[3] Chaudhri V K, Farquhar A, Fikes R, Karp PD, Rice JP (1998) Open Knowledge Base Connectivity 2.0.3. Technical Report KSL-98-06, Knowledge Systems Laboratory, Stanford, CA, http://www.ai.sri.com/ okbc/okbc-2-0-3.pdf.

[4] De Diego R (2001) Método de mezcla de catálogos electrónicos. Grade thesis, Facultad de Informática, Universidad Politécnica de Madrid. 2001

[5] Euzenat J (2004) An API for ontology alignment, in: Proceedings of the third International Semantic Web Conference (ISWC).

[6] Farquhar A, Fikes R, Rice J (1997) The Ontolingua Server: A Tool for Collaborative Ontology Construction. International Journal of Human Computer Studies. 46 (6), 707-727.

[7] Ganter B, Wille R (1997) Formal Concept Analysis: Mathematical Foundations. SpringerVerlag New York, Inc., Secaucus, NJ, USA Translator-C. Franzke.

[8] Gessler DD, Joslyn CA, Verspoor KM, Schmidt SE (2006) Deconstruction, re-construction, and ontogenesis for large, monolithic, legacy ontologies in semantic web service applications. Technical Report 06-5859, Los Alamos

[9] Giménez-Lugo GA, Amandi A, Sichman JS, Godoy D (2002) "Enriching Information Agents' Knowledge by Ontology Comparison: a Case Study", in the Proceedings of 8th Ibero-American Conference on Artificial Intelligence (IBERAMIA'02), Seville, Spain.

[10] Gómez-Pérez A, Fernández-López M, Corcho O (2003) Ontological Engineering. Springer-Verlag, London (United Kingdom).

[11] Gómez-Pérez A, Juristo N, Pazos J (1995) Evaluation and assessment of knowledge sharing technology. N.J. Mars (ed.). Towards Very Large Knowledge Bases. Knowledge Building and Knowledge Sharing. pp. 289-296, IOS Press.

[12] Gómez-Pérez A. (2003) Ontology Evaluation. Handbook on Ontologies, S. Staab and R. Studer, eds., Springer-Verlag, pp. 251-274.

[13] Gruber TR (1993) Toward principles for the design of ontologies used for knowledge sharing. Int. Workshop on Formal Ontology in Conceptual Analysis and Knowledge Representation. Padova, Italy. Kluwer Academic Publishers. Netherlands.

[14] Gruber TR (1995) Toward principles for the design of ontologies used for knowledge sharing, International Journal of Human-Computer Studies, v.43 n.5-6.

[15] Gruber TR (1993) A translation approach to portable ontology specifications. Knowledge Acquisition, 5: 199-220.

[16] Guarino N, Welty C (2000) A Formal Ontology of Properties. In: R. Dieng and O. Corby (eds.), Knowledge Engineering and Knowledge Management: Methods, Models and Tools. 12th Intnal. Conference, EKAW2000. Springer Verlag.

[17] Hyung Hwang S, Kim HG, Yang HS (2005) A FCA-based ontology construction for the design of class hierarchy. In: ICCSA (3). pp 827-835 
[18] IEEE 1996. IEEE Standard for Developing Software Life Cycle Processes. IEEE Std 1074-1995. IEEE Computer Society, New York.

[19] Kim IC (2004) FCA-based ontology augmentation in a medical domain. In: PAKM.

[20] Lopez-Pellicer F, Vilches-Blázquez LM, Nogueras-Iso J, Corcho O, Bernabé MA, Rodríguez AF (2007) Using a hybrid approach for the development of an ontology in the hydrographical domain. In: Proceedings of 2nd Workshop of COST Action C21 Towntology Ontologies for urban development: conceptual models for practitioners.

[21] Maedche A, Staab S (2001) Comparing Ontologies- Similarity Measures and a Comparison Study, Institute AIFB, University of Karlsruhe, Internal Report.

[22] Mizoguchi R, Vanwelkenhuysen J, Ikeda M (1995) Task ontology of reuse of problem solving knowledge. Towards Very Large Knowledge Bases: Knowledge Building \& Knowledge Sharing, pp. 46-59.

[23] Noy NF, Musen MA (2004) Using prompt ontology-comparison tools in the EON ontology alignment contest, in: Proceedings of the Third International Workshop Evaluation of Ontology-based Tools (EON).

[24] Noy NF, Fergerson RW, Musen MA (2000) The knowledge model of Protege-2000: Combining interoperability and flexibility. In: Dieng R, Corby O (eds) $12^{\text {th }}$ International Conference in Knowledge Engineering and Knowledge Management (EKAW’00). JuanLes-Pins, France. (Lecture Notes in Artificial Intelligence LNAI 1937) Springer-Verlag, Berlin, Germany, pp 17-32

[25] Rodríguez Pascual AF, García Asensio L (2005) “A fully integrated information system to manage cartographic and geographic data at a 1:25,000 scale". XXII International Cartographic Conference (ICC2005). A Coruña, Spain. ISBN: 0-958-46093-0

[26] Studer R, Benjamins VR, Fensel D (1998) Knowledge Engineering: Principles and Methods. IEEE Transactions on Data and Knowledge Engineering 25(1-2):161-197

[27] Uschold M, Grüninger M (1996) "Ontologies: Principles, Methods and Applications". Knowledge Engineering Review 11(2):93-155

[28] van Heijst G, Schreiber A, Wielinga B (1997) Using explicit ontologies in KBS development. Int. J. of Human-Computer Studies, 46(2/3):183-292.

[29] Vilches-Blázquez LM, Bernabé-Poveda MA, Suárez-Figueroa MC, Gómez-Pérez A, Rodríguez-Pascual AF (2007) "Towntology \& hydrOntology: Relationship between Urban and Hydrographic Features in the Geographic Information Domain”. In Ontologies for Urban Development. Studies in Computational Intelligence, vol. 61, pp. 73-84. Springer.

[30] Wang JZ, Ali F, Appaneravanda R (2005) A Web Service for Efficient Ontology Comparison. ICWS 2005: 843-844

[31] Wang JZ, Ali F (2005) An Efficient Ontology Comparison Tool for Semantic Web Applications. Web Intelligence 2005: 372-378

[32] Weinstein P, Birmingham W (1999) Comparing concepts in differentiated ontologies. In Proceedings of KAW-99.

[33] Zimmermann K. (2004) Ontology Comparison D19 v0.1. SW-Portal Working Draft. Available in http://www.deri.at/research/projects/sw-portal/2004/d19/v0.1/20040906/

[34] Dean M, Schreiber G (2003) OWL Web Ontology Language Reference. W3C Working Draft. http://www.w3.org/TR/owl-ref/

[35] McGuinness DL, van Harmelen F (2004) OWL Web Ontology Language Overview. W3C Recommendation. http://www.w3.org/TR/owl-features/

[36] Fonseca FT, Egenhofer MJ, Davis CA, Câmara G (2002) "Semantic Granularity in Ontology-Driven Geographic Information Systems". AMAI Annals of Mathematics and Artificial Intelligence. Volume: 36, 2002. Issue: 1-2, p. 121 - 151. 